See discussions, stats, and author profiles for this publication at: https://www.researchgate.net/publication/6577250

\title{
Selected eHealth Applications in Cyprus from the Training Perspective
}

Article in Methods of Information in Medicine · February 2007

DOI: 10.1055/s-0038-1628139 · Source: PubMed

CITATIONS

6 authors, including:

\section{Antonis Jossif}

"Paedi" Center for Specialized Pediatircs

13 PUBLICATIONS 43 CITATIONS

SEE PROFILE

Andreas Pitsillides

University of Cyprus

302 PUBLICATIONS 3,021 CITATIONS

SEE PROFILE

Some of the authors of this publication are also working on these related projects:

Project mEducator View project

Project IEEE Internet Computing editorial board View project

\section{READS}

54

C. S. Pattichis

University of Cyprus

431 PUBLICATIONS 5,663 CITATIONS

SEE PROFILE

Efthyvoulos C Kyriacou

Frederick University

174 PUBLICATIONS 2,436 CITATIONS

SEE PROFILE 
Accepted Special Issue of Methods of Information in Medicine Journal: Health and Medical Informatics Applications - Educational Aspects,

August 2005

Revised May 2006

\title{
Selected eHealth Applications in Cyprus from the Training Perspective
}

\author{
A. Jossif (1), C.S. Pattichis (2), M. Kyriakides (3), A. Pitsillides (2), E. Kyriacou (2), M. Dikaiakos \\ (2)
}

(1) Deparment of Paediatrics, Makarios Hospital, Nicosia, Cyprus

(2) Department of Computer Science, University of Cyprus, Cyprus

(3) Ministry of Health, Cyprus

Correspondence:

A. Jossif

Department of Paediatrics,

Makarios Hospital, Nicosia, Cyprus

email : ajossif@cytanet.com.cy 


\section{Summary}

Objectives: In this paper a review of selected eHealth applications in Cyprus is presented linked with their success or failure based on their training activities. Methods: The eHealth systems presented and their training activities include an update of the health information system (HIS) in the public hospitals, a medical system for emergency telemedicine (AMBULANCE and EMERGENCY-112 projects), a home monitoring system for cancer patients (DITIS), a satellite-based network in health-care applications (EMISPHER and HEALTHWARE projects), and the training activities of the Cyprus Society of Medical Informatics. Different methodologies for training were used ranging from classical approaches like train the trainers, using demo cases followed by personal training, group training, workshops, to more recent methodologies based on eLearning sessions including teleconsultations. Results: The training was carried out successfully in all cases. However, not all eHealth systems were put into practise successfully, mainly for reasons not related to training. Conclusions: It is anticipated that this paper will promote the importance of these applications and their training activities as well as help in the spin off of others thus enabling the offering of a better service to the citizen.

Key-words : eHealth, Health Telematics, Medical Informatics, eLearning, Training 


\title{
Selected eHealth Applications in Cyprus from the Training Perspective
}

\author{
A. Jossif (1), C.S. Pattichis (2), M. Kyriakides (3), A. Pitsillides (2), E. Kyriacou (2), M. \\ Dikaiakos (2)
}

(1) Deparment of Paediatrics, Makarios Hospital, Nicosia, Cyprus

(2) Department of Computer Science, University of Cyprus

(3) Ministry of Health, Cyprus

\section{Introduction}

Rapid advances in information technology and telecommunications and their convergence (telematics) are leading to the emergence of a new type of information infrastructure that has the potential of supporting an array of advanced services for healthcare, that is commonly called eHealth. In the constant competition for resources in the health sector these new eHealth technologies and applications would not stand much chance if they were not intended to improve the quality of care, directly or indirectly, to help in the cost-containment and better management of health sector, and to increase competitiveness of the medical informatics industry, including health telematics [1]. Cyprus, although at its early stages in eHealth systems, is actively participating in a number of promising applications covering a spectrum of healthcare processes and services. The objective of this paper is to provide a snapshot of selected eHealth applications, and to discuss their training activities leading to their success or failure.

In Cyprus there was a major effort to introduce a health information system (HIS) in the public hospitals that is considered to have been a partial failure. At the same time several telematics pilot projects were funded and developed but only a few are being currently used and are considered to be successful. We will present the effort to introduce the HIS (the outcome of this project was not what it was expected), and we will also discuss the efforts for a new initiative to introduce a new HIS for the public hospitals which is currently underway. Also, three health telematics projects are presented related to emergency telemedicine, home monitoring, and a satellite-based network that can be used for health-care applications. The training aspects of all these projects are presented as well as the reasons for their success or failure. Furthermore, the training activities of the Cyprus Society of Medical Informatics are presented.

\section{The Health Information System of the Ministry of Health [2]}

The introduction of the Governmental Health Information System commenced in 1989 as a co-project between the Ministry of Health of the Republic of Cyprus and the East Mediterranean Region Office (EMRO) of the World Health Organisation (WHO). Firstly, the Patient Administration System (PAS) module was implemented. The pilot commenced in 1991 at Archbishop Makarios III Hospital in Nicosia and was successfully completed in 1993. WHO evaluated the results of the pilot implementation at a regional conference in Limassol in June 1993. Subsequently, the Ministry of Health in collaboration with the Department of Information Technology Services proceeded with the PAS implementation in the other three district General Hospitals which was completed in March 1999. The PAS module was subsequently introduced at three Rural Hospitals. Other modules besides the patient's personal and demographic data that have been introduced at the four Hospitals are: (i) Doctor's register, (ii) Scheduling for the outpatient department, (iii) outpatient encounters and (iv) in-patient register. 
The initial training of users was delayed in relation to the deployment of the system at the first site (Makarios Hospital) which delayed the whole process. The training started with the first group of administrative staff and the nursing personnel followed. To date 760 employees have been trained from all the hospitals, $80 \%$ of them are nursing personnel, about $20 \%$ administrative staff and a small number are medical staff.

In June 2003, the Ministry of Health and the Department of Information Technology Services of the Ministry of Finance have published an expression of interest (EOI) document for the establishment of a turnkey solution of a ready-made application software for an Integrated Health Care Information System (IHCIS). The system will cover the clinical, administrative and financial activities of both the New Nicosia General Hospital and the New Famagusta General Hospital. The envisioned system will have the capability for a full electronic patient record, be web-enabled and support the vision of the Ministry of Health for paperless and film less hospitals. The IHCIS consists of the following thirteen modules: Patient Administration, Electronic Health Care Record, Hospital Order Entry, Clinical Laboratory, Radiology/PACS, Billing, Stock Control, Prescription Management, Personnel Management, Blood Bank, Health Smart Card, Histopathology, and Coding and Classification of clinical terms. At present the evaluation of the tenders is in progress, and the deployment of the first phase is expected to be completed by the mid of 2006 .

The tenderers shall propose, design, and implement appropriate training packages for each of the modules of the IHCIS listed above as well as an introductory course regarding the overall system functionality shall be provided to all users. The tenderer shall provide a detailed plan of the training courses, outlining and justifying the sequence and frequency of the courses, and shall describe the way and practice to ensure a successful training outcome. The tenderer shall include detail information for all offered training courses including the following: course name, duration (hrs), location, aims of the training course, course outline, instructors qualifications, and offered certification at the end of the training course.

A detailed list of prospective users has already been prepared for training that includes around a 1000 and 250 users for the New Nicosia and New Famagusta Hospitals respectively. A Train the Trainers methodology will be followed, where a group of 10 Expert Users will be trained and will be responsible for further End Users training and first line support in each of the hospitals.

The whole project is expected to take 12 months. It should be noted that this time schedule is considered to be optimistic by a number of officers in the Ministry of Health, based on the rather limited information technology experience of the personnel of the hospitals. However, the plans at present are to proceed as originally scheduled.

Furthermore, the University of Cyprus, in collaboration with the Ministry of Health and the Pafos General Hospital has recently been awarded a new INTERREG III B Archimed Program project entitled "A Mediterranean Research and Higher Education Intranet in Medical and Biological Sciences" [3]. The aim of this project is the development of a medical educational/research intranet between higher education and research institutions from Greece, Italy and Cyprus, to support the undergraduate educational program, graduate practice and residency and to promote research collaborations by: (i) dissemination of webbased lectures and seminars, covering advanced scientific topics and up-to-date technologies, and (ii) promotion of research collaborations through web-based shared workspaces.

\section{Emergency Telemedicine: the AMBULANCE and EMERGENCY-112 Projects [4]-[6]}

The availability of prompt and expert medical care can meaningfully improve health care services at understaffed rural or remote areas. The provision of effective emergency telemedicine and home 
monitoring solutions are the major fields of interest of AMBULANCE HC1001and EMERGENCY-112 HC4027 projects that were partially funded by the European Commission / DGXIII Telematics Application Programme.

The aim of the AMBULANCE project was the development of a portable emergency telemedicine device that supports real-time transmission of critical biosignals as well as still images of the patient using the GSM link. This device can be used by paramedics or not specialized personnel that handle emergency cases, in order to provide telediagnosis, long distance support and directions from expert physicians located at an emergency coordination centre or a specialized hospital.

The system can be used for different cardiovascular or severe injuries cases. It comprises of two different modules: (i) the mobile unit, which is located in an Ambulance vehicle near the patient, and (ii) the consultation unit, which is located at the hospital site and can be used by the experts in order to give directions.

EMERGENCY-112, which was the extension of the AMBULANCE project, aimed the extension of the system to an integrated system which would be able to operate over several communication links (Satellite, GSM, POTS, ISDN, LAN etc. ) and for different cases (ambulance emergencies, rural health center or any other remotely located health center support, navigating ships support and home monitoring). In EMERGENCY-112, emphasis was given to maximizing the system's future potential application, through the utilization of different links (both fixed and wireless), as well as through the increase of the overall system's usability, focusing on advanced user-interface and ergonomics. The system comprises of two different modules: (i) The patient unit which is the unit located near the patient. This unit can operate automatically and has several operating features (depending on the case used). (ii) The physician's unit which is the unit located near the expert doctor. This unit can be either fixed or mobile depending on the place where the expert doctor is located. Diagnostically important data, like ECG, blood pressure, heart rate, oxymetry, temperature, etc., are collected via a biosignal monitor connected to a portable computer at the mobile site and are transmitted through the mobile telephony network (i.e satellite, GSM or UMTS) to the hospital site. Still images of the patient's position and state are captured through a small camera and transmitted. The specialist at the hospital site can observe the signals in real-time, view the images of the patient and mark some interesting areas (whiteboadring), a marking that appears simultaneously at the mobile screen. Thus, he is able to assess the severity of the emergency and through a bidirectional voice communication link can instruct the paramedic how to handle the case. The system is supported by a multimedia database, which stores all information available from the time the system is initialized until the arrival of the patient at the hospital.

The system was evaluated and verified in Cyprus, Greece, Italy and Sweden. Each pilot recorded certain time indicators, such as time-to-transportation, time-to-start-treatment, time-to-stabilization, etc., in a total of 100 cases in which the system was used and in another 100 cases without using the system, in order for comparative results to be deduced. It was shown that the system provides significant support to the early and specialized pre-hospital patient management and to emergency case survival. The diagnosis at the scene of an emergency, as well as the handling of the case, was substantially improved through on-line access to medical specialists, which decreased the time to make the first diagnosis and start the appropriate treatment. Severe or multiple trauma patients were better assessed, while the electronic registration of the patient's data freed the ambulance personnel of any paper work and helped devoting more time on real emergency care.

Training of users was performed on each site where the base stations and clients of the system were installed. The age of the users ranged from 25 to 50 years old. Users were divided into experienced and non experienced computer users. Training was initially performed using demo cases. This was essential since the system's main purpose was the support of emergency medical cases. Initial training time for each experienced user was about 30 minutes whereas for not experienced users it was about 1 hour. 
Furthermore the use of the system initially was performed in collaboration with the technical people, thus allowing users to continue their training. A user manual with instructions and installation disks was supplied to the sites.

The results from the system use (except from the initial evaluation phase) were not at the success level we initially expected. In general the system was introduced and used in daily routine only in one hospital which was established when the system was created. This is the Interbalkan Hospital in Thessaloniki Greece. The emergency health care department was created at the time when the system was finished; the people of the department were trained from the beginning and the system became part of their daily routine. The same department was supporting two isolated areas in the northern part of Greece; a village called "Zoni" and a small island called "Agios Efstratios". Unfortunately at the other sites in Greece, Italy, and Sweden where the system was installed, the departments were already established and the use of the system was initially delaying the process since many of the users were not computer experts. The final result was the use of the system rarely.

In Cyprus, although the system was evaluated successfully at the Department of Accident and Emergency at the Nicosia General Hospital, connecting a rural health centre and an ambulance, it has not yet been set in routine operation. However, this project will now be revitalized based on a new INTERREG III B Archimed Program project entitled "An INTEgrated broadband telecommunication pilot teleservicesplatform for improving health care provision in the Region of MEDiterranean" that has recently been awarded to the University of Cyprus and the Pafos General Hospital [7]. The aim of this project is to develop a platform that will enhance the provision of medical services for both citizens and traveller's in remote/isolated regions of southeast Mediterranean, and on board ships travelling across it. The platform will support ECG and vital signal processing, as well as, ultrasound imaging and video conference functionality.

\section{DITIS: Home Healthcare of Cancer Patients [8]-[11]}

DITIS ( $\triangle \mathrm{ITH} \Sigma$, in Greek, stands for: Network for Home HealthCare Collaboration) is a system that supports Virtual HealthCare Teams dealing with the home-healthcare of cancer patients in Cyprus. DITIS was originally developed with a view to address the difficulties of communication and continuity of care between the home health care multidisciplinary team (of the Pancyprian Association of Cancer Patients and Friends, PASYKAF) and between the team and the oncologist often over $100 \mathrm{kms}$ away. DITIS has through its database and possibility of access via mobile or wire line (computers) offered much more than improved communication. Its flexibility of communication and access to the patient's history and daily record at all times and from anywhere (e.g. home, outpatients, or even during emergency admission) has offered the team a continuous overall assessment and history of each symptom. DITIS supports the creation, management and co-ordination of virtual healthcare teams, for the continuous treatment of the patient at home. Thus it has offered improved quality of life to the patient, for example by offering the nurses the possibility of immediate authorisation to change prescription via mobile devices and the oncologist the possibility of assessment and symptom control without necessarily having to see the patient.

DITIS was initiated in 1999 as a two-year research project and has since been funded by a number of organizations: the Cyprus Research Promotion Foundation, the University of Cyprus, and the Cyprus Association of Cancer Patients and Friends (PASYKAF). DITIS has also been supported by the Cyprus Telecommunications Authority (CYTA), Cambridge Microsoft Research Labs, NetU consultants Ltd, WinMob Technologies Ltd, Ericsson (through S.A. Petrides Ltd), and the Cyprus Development Bank (CDB). Currently, DITIS supports the activities of PASYKAF, who offer home-care services for cancer patients in Cyprus. Work is underway for a second phase of the project, leading to pan Cyprian deployment and commercialization. 
Among its international successes, DITIS was a finalist in the eHealth Ministerial conference in 2003 (ranked among the best 25 eHealth projects out of 179 EU projects), ranked 7th in the 2003 World Summit Award (out of 89 entries in e-content from 89 countries), and was the only non-IST funded project participating in the cluster project MEMO due to its mobile collaboration aspect. Currently, DITIS is the subject of an extensive cost-benefit evaluation by the Association of Chartered Certified Accountants (ACCA), as commissioned by the EC. The purpose of the case study is to show economic benefits from adopting ICT in healthcare, beyond the undisputable social and medical benefits. The results will be presented during the 2005 eHealth Ministerial Conference and at the EU parliament to stir debate in support for eHealth.

DITIS is at present being deployed, for its healthcare collaboration and patient management aspects, in the context of two EU funded e-TEN market validation projects (HealthService24 [11] and LinkCare). Both projects involve trials for cardiac patient monitoring which are in progress at the LITO polyclinic. They feature the first adaptations of DITIS to be used in an acute care environment (as opposed to the chronic patient environment for cancer patients).

The system was successfully implemented, tested, and deployed at PASYKAF due to the pragmatic approach that it follows in supporting the home health care team and due to a highly motivated team that pays special attention to the needs of the end-users.

Training in support of DITIS was provided to all the healthcare staff including doctors, nursing personnel, psychologists, social workers, and administrative staff. The age of these personnel ranged from 23 to 55 years old, and they could be easily separated into two groups: (i) experienced and (ii) non-experienced users. For experienced users, a 4 hours-long training session was prepared, covering mainly the application software (30 minutes about the DITIS project, 30 minutes introduction to the use of computers in medical applications, one hour about overall DITIS system functionality and usage, and one hour about the specific functionality for each group of users). For non-experienced users, 16-24 hours training was provided, beginning with basic computer skills training (mouse, keyboard, main windows applications, and internet) and then covering mobile devices and the application software.

Training was initially organized for groups of four (for personnel with the same specialization), and then where needed was followed-up with one-to-one training, targeting each individual's needs. The initial training included a general presentation of the functionalities of the system, as well as the functionalities relating to each individual's discipline (role). The individual training sessions were of about 3 hours long. They included:

- Introduction to the computer basics, if needed (20 to 30 minutes).

- Introduction to the DITIS program. This was also covered in the group training, but it gave a chance to be more interactive and specific in this case (about 10 minutes).

- A generalized presentation of all the functionalities of the system, with emphasis on user role (about 1 hour).

- A comprehensive hands-on use of the system, via a structured approach (i.e. by adding a new patient and progressively introducing other functionalities of the system, as for example the appointments, pain and symptom diaries, and so on) (about 2 hours).

A system manual was prepared and given to the users, supplemented by a support team, which provided support as needed, dependant on their availability as there is no dedicated support personnel (the system designers and developers double up in this role). Basically, within our constraints, there was every effort to provide continuous user support relating to the use of the system.

A few points worth noting are: 
- Users were encouraged to install the system in their home PCs (the majority have a home PC), which helped to use the system whenever it was more suitable to them.

- Users were given the option to request another training session if they felt uneasy about using the system (this happened in very few cases).

- Language turned out to be a problem. PASYKAF employs a large number of non- Greekspeaking nurses (permanent residents of Cyprus) and a number of Greek- speaking nurses (some not conversant in English). This was particularly problematic, especially in group training sessions with both Greek-speaking and English-speaking participants. Note that the interface is currently available only in English, but plans are underway to offer it in Greek also.

Based on our experiences, we see that the approach undertaken to offer both group and individual training has paid off. After the training sessions, system usage increased dramatically. The continuous, ondemand, customised training provided was considered successful for the needs of the users of DITIS.

In summary, DITIS is adopted today, mainly because so much was given to supporting the end users in their efforts to learn the technology. The commitment of the entire team has been enormous in providing different types of training based on the real needs of users. DITIS is a success not only because it is an innovative product (there are many innovative products that never get deployed), but because the time and effort was taken to train the users, and a continuous effort is still on-going to support them. Eventually, we hope to have users within the adopting organization who become trainers themselves.

\section{Satellite Networks for Healthcare: from Emispher to Healthware [12]-[15]}

The feasibility of satellite-based networks in health-care applications has been investigated in the context of the EMISPHER (Euro-Mediterranean Internet-Satellite Platform for Health, Education and Research) project, which was co-funded by the European Commission in the frame of the program EUMEDIS promoting cooperation between Mediterranean countries. The goal of the EMISPHER project was to establish a broadband satellite network and to demonstrate its use in a number of different health-carerelated scenarios, ranging from eLearning and real-time tele-consultation to medical assistance. The project lasted for two years; following a slow start, which was attributed to administrative and contractual difficulties, the EMISPHER consortium established a satellite network with 10 sites in respective countries (Casablanca, Algiers, Clermond Ferrand, Berlin, Tunis, Palermo, Athens, Istanbul, Nicosia, and Cairo). Each site was equipped with a VSAT terminal and a telemedicine workstation, WoTeSa (Workstation for Telemedical Applications via Satellites), which consisted of a PC with two Pentium processors, and high quality video camera and frame-grabber, and the WinVicos communication software. WoTeSa was also used to run HealthE, a Java program providing electronic management of the Medical Records required in Medical Assistance scenarios [12]. Eutelsat provided the satellite bandwidth; access to the satellite network and bandwidth reservation was managed with the MedSky software developed by Telemedicine Technologies SA [14] and installed on WoTeSa.

The EMISPHER network was used in Cyprus in a number of very successful trials that involved connections between two or more partners implementing real-time eLearning sessions and teleconsultation. In particular, the platform was used on August 27, 2004, in a seminar on eLearning in the Intensive Care Unit, which was organized by Dr. Th. Kyprianou, Nicosia General Hospital, and Dr. Daniella Filli, Istituto Mediterraneo per i Trapianti e Terapie ad Alta Specializzazione (ISMETT), Palermo. The proceedings of the seminar were broadcasted through the EMISPHER platform to participants in both Nicosia and Palermo (doctors, nurses, and IT professionals). EMISPHER was also demonstrated with real-time telemedicine sessions between Cyprus and other EMISPHER partners (in Berlin, Tunis, and Algiers), to medical doctors from different medical institutions of Cyprus, including the Department Radiology of the Nicosia General Hospital and the Bank of Cyprus Oncology Centre. 
Currently, the EMISPHER platform is being integrated in the new tele-education room of the Department of Computer Science, University of Cyprus. The platform will be used in a number of eLearning events that are planned by the Intensive Care Forum of Cyprus. In continuation to EMISPHER, the Deparment of Computer Science at the University of Cyprus (High-Performance Computing Systems Lab) is participating to the HEALTHWARE (HEALTH care services over Wide AREas) Integrated Project funded by the European Union under Framework Programme 6, Priority 4 (Aeronautics and Space) and coordinated by Alcatel [15]. The goal of HEALTHWARE is to boost the deployment of DVB-RCS (Digital Video Broadcasting / Return Channel via Satellite) technology on a large scale. At the time of this writing, the University of Cyprus is actively collaborating with the Nicosia General Hospital (Intensive Care Unit) in order to define the scenarios and the infrastructure that will be deployed in Cyprus, in the context of HEALTHWARE.

\section{Cyprus Society of Medical Informatics - Training Activities [16]}

The Cyprus Society of Medical Informatics (CSMI) was established in 1997 and it has 180 members, mainly physicians. CSMI is a member of the European Federation of Medical Informatics (EFMI). Since its establishment, it has been involved in training of its members on computer fundamentals and office applications. Several formal classes and lectures are offered annually.

Also, in collaboration with the University of Cyprus, it participated in the EU Leonardo da Vinci project Health Telematics Training Network (HEALTHNET, 1997-2000) [2]. This project prepared the necessary material for training the physicians on teleworking. The CD prepared covered the topics of internet fundamentals, web browsing, and health telematics applications. The material included extensive hands on experience, and it was taught in four sessions, four hours each. A total of 60 physicians were trained using the HEALTHNET methodology and material.

Furthermore, major additional activities of the society included the organization of the Special Topic Conference "The e-volution of IT in the Health Care Systems", together with the Workshop on "Natural Language Processing in Biomedical Applications", in collaboration with EFMI (corresponding WG) in March 2002. Also, CSMI, in collaboration with the University of Cyprus and the Greek Health telematics forum organized a day meeting on eHealth, in April 2002. In November of 2003 CSMI with the support of EFMI and the University of Cyprus organized the conference "Electronic Health Records and Telemedicine: Implications for Better Clinical Practice". The society is currently planning a workshop on Electronic Patient Records for Small Clinics, in 2006.

\section{Concluding Remarks}

In this paper a review of selected eHealth applications in Cyprus was presented linked with their success or failure based on their training activities. Different methodologies for training were used ranging from classical approaches like train the trainers, using demo cases followed by personal training, group training, workshops, to more recent methodologies based on eLearning sessions including teleconsultations. The training was carried out successfully in all cases. However, not all eHealth systems were put into practise successfully, mainly for reasons not related to training.

Cyprus should accelerate its pace in eHealth services for the benefit of the whole health care sector. A critical factor in achieving this is training of the physicians, the paramedical and administrative staff in emerging information technologies in healthcare. Training of these personnel should include concepts like electronic patient record, web-based systems, security, teleworking, and other. Linked with the training, there should be a 24 hours support of the medical staff in the hospital, clinical systems, or home care under operation. Furthermore, based on the recommendations of the WHO report on eHealth Tools \& Services [17], Cyprus should invest more on eLearning methods in health sciences, provide wider 
access to digital libraries and information about evidence-based research within the eHealth domain, and help in the establishment of a network to share experiences internationally. These actions should be promoted taking into consideration recent trends in health informatics education and training based on the introduction of new technologies, including the electronic patient record and ePrescription [18], as well as hitech imaging, robotics, and genomics and proteomics applications [19].

Finally, Cyprus should prescribe its national action plan in eHealth, aligned with the recently announced EU eHealth action plan [20]. This national plan should prescribe the road map leading to the wider spread of information and communication technologies enabling the faster implementation of electronic health records, patient identifiers and health cards, and the faster rollout of high speed internet access to enable optimum interactions among health care professionals and with the general public [21]. It is hoped that these efforts will be materialized, thus enabling the offering of a better service to the citizen.

\section{Acknowledgements}

This study was partly supported through the EU European Regional Development Fund, INTERREG III B Archimed Program, projects: i. "A Mediterranean Research and Higher Education Intranet in Medical and Biological Sciences", and ii. "An INTEgrated broadband telecommunication pilot teleservicesplatform for improving health care provision in the Region of MEDiterranean", June 2006 - December 2007.

\section{References}

[1] Iacovides I, Pattichis CS, Schizas CN. Editorial special issue on emerging health telematics applications in Europe. IEEE Trans. on Inform. Technol. in Biom. 1998; 2; No. 3: 2-8.

[2] Pattichis CS, Schizas CN, Andreou A. Healthcare telematic applications in Cyprus. Methods of Information in Medicine 2002; 41: 376-381.

[3] IntraMEDnet. Web Site: http://www2.cs.ucy.ac.cy/intramednet (last accessed November 2006).

[4] Pavlopoulos S, Kyriakou E, Berler A, Dembeyiotis S, Koutsouris D. A novel emergency telemedicine system based on wireless communication technology - AMBULANCE. IEEE Trans. on Inform. Technol. in Biomed. - Special Issue on Emerging Health Telematics Applications in Europe 1998; 2; No. 4: 261-267.

[5] Pattichis CS, Kyriacou E, Voskarides S, Pattichis MS, Istepanian R, Schizas CN. Wireless telemedicine systems: An overview. IEEE Antennas \& Propagation Magazine 2002; 44, No. 2 , 143-153.

[6] Kyriacou E, Sotiris P, Berler A, Neophytou M, Bourka A, Georgoulas A, Anagnostaki A, Karayiannis D, Schizas C, Pattichis C, Andreou A, Koutsouris D. Multi-purpose HealthCare telemedicine systems with mobile communication link support. BioMedical Engineering OnLine, http://www.biomedical-engineering-online.com/start.asp 2003; 2, No. 7.

[7] Mougiakakou SG, Papadopoulos H, Telonis P, Konnis G, Nikita KS, Koutsouris D. An Integrated Telecommunication Pilot Platform for the Provision of Telematic Health Care Services. CD-ROM Proceedings of the 5th International IEEE EMBS Special Topic Conference on Information Technology Applications in Biomedicine, 26-28 October, 2006, Ioannina, Greece, 6 pages.

[8] DITIS. Web Site: www.ditis.ucy.ac.cy (last accessed November 2006).

[9] Pitsillides A, Pitsillides B, Samaras G, Dikaiakos M, Christodoulou E, Andreou P, Georgiadis D. DITIS: A Collaborative Virtual Medical Team for Home Healthcare of Cancer Patients. In: M-Health: Emerging Mobile Health Systems, RH Istepanian, S Laxminarayan, CS Pattichis, editors, Springer Science, 2006; 247-266. 
[10] Pitsillides A, Samaras G, Pitsillides B, Georgiades D, Andreou P, Christodoulou E. Virtual Collaborative Healthcare Teams for Home Healthcare. Journal of Mobile Multimedia (JMM), special issue on Advanced Mobile Technologies for Health Care Applications 2006; 2, No.1, 23-36.

[11] HealthService 24. Web Site: www.healthservice24.com (last accessed November 2006).

[12] EMISPHER Project. Web Site: http://www.emispher.org (last accessed November 2006).

[13] Grashew G, Roelofs TA, Rakowsky S, Schlag PM. GALENOS as interactive telemedical network via satellite. In: Optical Network Design and Management, X Ren, T Aoyama, editors, Proc. of SPIE 2001; Vol. 4584, 202-204.

[14] Telemedicine Technologies S.A. Web Site: http://www.tentelemed.com (last accessed July 2005).

[15] HEALTHWARE Project. Web Site: http://healthware.alcatel.net (last accessed July 2005).

[16] Cyprus Society of Medical Informatics. Web Site: http://www.csmi.org.cy/ (last accessed July 2005).

[17] eHealth TOOLS \& SERVICES, Needs of the Member States, Report of the WHO Global Observatory for eHealth, WHO, 2006.

[18] Stepankova O, Engova D. Professional competence and computer literacy in e-age, focus on healthcare. Methods of Information in Medicine 2006; 45 3: 300-304.

[19] Moehr JR. Where to in the next ten years of health informatics education? Methods of Information in Medicine 2006; 45 3: 283-287.

[20] e-Health - making healthcare better for European citizens: An action plan for a European e-Health Area, Communication from the Commission to the Council, the European Parliament, the European Economic and Social Committee and the Committee of the Regions, Commission of the European Communities, Brussels, 30.4.2004, COM (2004) 356 final. Web Site: http://europa.eu.int/information_society/doc/qualif/health/COM_2004 0356_F EN_ACTE.pdf, (last accessed July 2005).

[21] eHealth: Better Healthcare for Europe. Web Site: http://europa.eu.int/information_society/activities/health/index_en.htm, (last accessed July 2005). 